\title{
MODULES WITH SEMI-LOCAL ENDOMORPHISM RING
}

\author{
DOLORS HERBERA AND AHMAD SHAMSUDDIN
}

(Communicated by Ken Goodearl)

\begin{abstract}
We use the concept of dual Goldie dimension and a characterization of semi-local rings due to Camps and Dicks (1993) to find some classes of modules with semi-local endomorphism ring. We deduce that linearly compact modules have semi-local endomorphism ring, cancel from direct sums and satisfy the $n$th root uniqueness property. We also deduce that modules over commutative rings satisfying $A B 5^{*}$ also cancel from direct sums and satisfy the $n$th root uniqueness property.
\end{abstract}

Let $R$ be an associative ring with 1 and let $M$ be a right unital $R$-module. A finite set $A_{1}, \ldots, A_{n}$ of proper submodules of $M$ is said to be coindependent if for each $i, 1 \leq i \leq n, A_{i}+\bigcap_{j \neq i} A_{j}=M$, and a family of submodules of $M$ is said to be coindependent if each of its finite subfamilies is coindependent. The module $M$ is said to have finite dual Goldie dimension if every coindependent family of submodules of $M$ is finite. It can be shown that, in this case, there is a maximal coindependent family of submodules of $M$. If this set is finite, then its cardinality (denoted by $\operatorname{codim}(M))$ is uniquely determined and is called the dual Goldie dimension of $M$. If this set is infinite we set $\operatorname{codim}(M)=\infty$ and say that $M$ has infinite dual Goldie dimension. A module with dual Goldie dimension 1 is said to be hollow, and a cyclic hollow module is said to be local . We have

$$
\begin{gathered}
\operatorname{codim}\left(M_{1} \oplus M_{2}\right)=\operatorname{codim}\left(M_{1}\right)+\operatorname{codim}\left(M_{2}\right), \\
\operatorname{codim}(M / N) \leq \operatorname{codim}(M) \text { for every submodule } N \text { of } M, \\
\operatorname{codim}(M / N)=\operatorname{codim}(M) \text { if } N \text { is a small submodule of } M, \\
\operatorname{codim}(M)=0 \text { if and only if } M=0 ;
\end{gathered}
$$

refer to [10] and [20] for details concerning the dual Goldie dimension.

A ring $R$ with Jacobson radical $J(R)$ is said to be semi-local if $R / J(R)$ is a semi-simple ring. Semi-local rings are characterized as those rings with finite dual Goldie dimension. Note that for a semi-local ring $R$,

$$
\operatorname{codim}\left(R_{R}\right)=\text { length of the right } R \text {-module } R / J
$$

and so $\operatorname{codim}\left(R_{R}\right)=\operatorname{codim}\left({ }_{R} R\right)$; this common value is denoted by $\operatorname{codim}(R)$.

Received by the editors December 27, 1993 and, in revised form, May 2, 1994.

1991 Mathematics Subject Classification. Primary 16D70, 16L30, 16S50; Secondary 16P60. 
Denote by $\operatorname{dim}(M)$ the Goldie dimension of $M$ and by $\mathrm{U}(R)$ the group of units in the ring $R$. Camps and Dicks recently proved the following characterization of semi-local rings.

Theorem 1 (Camps and Dicks [3, Theorem 1(e)]). A ring $R$ is semi-local if and only if there exist an integer $n$ and a function $d: R \rightarrow\{0, \ldots, n\}$ satisfying the conditions:

(1) for any $r, s \in R, d(r-r s r)=d(r)+d(1-r s)$,

(2) $d(r)=0$ if and only if $r \in \mathrm{U}(R)$.

Moreover, it follows in this situation that $\operatorname{codim}(R)=\operatorname{dim}(R / J(R)) \leq n$.

Recall that a ring $R$ has 1 in its stable range if whenever the equation $a x+b=1$ has a solution for $x$ in $R$, there exists $c \in R$ such that $a+b c \in$ $U(R)$. A right $R$-module $M$ cancels from direct sums if for any right $R$ modules $A$ and $B, M \oplus A \cong M \oplus B$ implies $A \cong B$.

By a result of Evans [6, Theorem 2], if 1 is in the stable range of the endomorphism ring of a module $M$, then $M$ cancels from direct sums. Bass in [2] proves that a semi-local ring has 1 in its stable range, and hence a module whose endomorphism ring is semi-local cancels from direct sums.

It has been recently proved by Facchini, Herbera, Levy and Vamos [7] that if $M$ and $N$ are modules for which the endomorphism rings End $M$ and End $N$ are semi-local, then $M^{n} \cong N^{n}$ for $n \in \mathbb{N}$ implies that $M \cong N$. This latter property is called the $n$th root uniqueness property.

We summarize these results in the following theorem.

Theorem 2. Let $R$ be a ring and $M$ a right $R$-module with semi-local endomorphism ring. Then 1 is in the stable range of the endomorphism ring of $M, M$ cancels from direct sums and $M$ satisfies the $n$th root uniqueness property.

In this paper we use Theorem 1 and the concept of dual Goldie dimension to find classes of modules whose endomorphism rings are semi-local. Our main result is Theorem 3 which contains the result of Camps and Dicks [3, Theorem 5] and has consequences for quasi-projective modules (Corollary 4), linearly compact modules (Corollary 5) and modules satisfying $A B 5^{*}$ and for which the number of non-isomorphic simple subfactors is finite (Corollary 7). In general the endomorphism ring of a right $R$-module $M$ satisfying $A B 5^{*}$ is not semilocal, but we can show that if $R$ is commutative, the endomorphism ring of $M$ is a product of semi-local rings, hence the conclusions of Theorem 2 are still valid for $A B 5^{*}$ modules over commutative rings (Corollary 9).

Example 10 (1) shows that any ring that can be embedded in a local ring can be realized as the endomorphism ring of a local module over some ring; this contrasts with the situation for quasi-projective modules (see Corollary 4), or with the situation for commutative or right noetherian rings (see the remarks preceding Corollary 4).

All our results seem to indicate that there is a close relation between having semi-local endomorphism ring and having finite dual Goldie dimension. However Example 10 (2) shows that there exist cyclic modules with semi-local endomorphism ring whose dual Goldie dimension is not finite.

We denote the endomorphism ring of the right $R$-module $M$ by $\operatorname{End}_{R}(M)=$ $\operatorname{End}(M)$. 
Theorem 3. Let $R$ be a ring and $M$ a right $R$-module.

(1) (Camps and Dicks [3, Theorem 5]) If $M$ has finite Goldie dimension and every injective endomorphism of $M$ is bijective, then the endomorphism ring of $M$ is semi-local and

$$
\operatorname{codim}(\operatorname{End}(M))=\operatorname{dim}(\operatorname{End}(M) / J(\operatorname{End} M)) \leq \operatorname{dim}(M) .
$$

(2) If $M$ has finite dual Goldie dimension and every surjective endomorphism of $M$ is bijective, then the endomorphism ring of $M$ is semi-local and

$$
\operatorname{codim}(\operatorname{End}(M))=\operatorname{dim}(\operatorname{End}(M) / J(\operatorname{End} M)) \leq \operatorname{codim}(M) .
$$

(3) If $M$ has finite dual Goldie dimension and finite Goldie dimension, then the endomorphism ring of $M$ is semi-local and

$$
\operatorname{dim}(\operatorname{End}(M) / J(\operatorname{End} M)) \leq \operatorname{dim}(M)+\operatorname{codim}(M) .
$$

Proof. If $f$ and $g$ are endomorphisms of $M$, then

$$
\operatorname{ker}(f-f g f)=\operatorname{ker}(f) \oplus \operatorname{ker}(1-g f),
$$

for it is clear that $\operatorname{ker}(f) \cap \operatorname{ker}(1-g f)=0$ and for any $x \in \operatorname{ker}(f-f g f)$, $x=g f(x)+(1-g f)(x)$ where $g f(x) \in \operatorname{ker}(1-g f)$ and $(1-g f)(x) \in \operatorname{ker}(f)$.

Dually,

$$
\operatorname{coker}(f-f g f) \cong \operatorname{coker}(f) \oplus \operatorname{coker}(1-f g)
$$

which holds because

$$
M=\operatorname{im}(f g)+\operatorname{im}(1-f g)=\operatorname{im}(f)+\operatorname{im}(1-f g)
$$

and

$$
\operatorname{im}(f-f g f)=\operatorname{im}(f) \cap \operatorname{im}(1-f g) .
$$

The endomorphism $f$ induces isomorphisms between $\operatorname{ker}(1-g f)$ and $\operatorname{ker}(1-f g)$, and between coker $(1-g f)$ and $\operatorname{coker}(1-f g)$.

To prove (1) let $n=\operatorname{dim}(M)$, define $d_{1}: \operatorname{End}(M) \rightarrow\{0, \ldots, n\}$ by $d_{1}(f)=\operatorname{dim} \operatorname{ker}(f)$ and set $d=d_{1}$. To prove (2) let $m=\operatorname{codim}(M)$, define $d_{2}: \operatorname{End}(M) \rightarrow\{0, \ldots, m\}$ by $d_{2}(f)=\operatorname{codim} \operatorname{coker}(f)$ and set $d=d_{2}$. To prove (3) set $d=d_{1}+d_{2}: \operatorname{End}(M) \rightarrow\{0, \ldots, n+m\}$. In each of the three cases $d$ satisfies the conditions of Theorem 1 and the result is now clear.

Camps and Dicks use Theorem 3 (1) to prove that artinian modules have semi-local endomorphism rings [3, Corollary 6] since for an artinian module any injective endomorphism is bijective.

Following Goodearl [9] we say that a ring $R$ is right repetitive if for any elements $a, b \in R$ the right ideal $I=\sum_{i \geq 0} a^{i} b R$ is finitely generated. Right repetitive rings include commutative rings, matrices over commutative rings and right noetherian rings. Goodearl in [9] shows that $M_{n}(R)$ is right repetitive for any $n \geq 1$ if and only if any surjective endomorphism of a finitely generated module $M$ is an isomorphism. Thus if $M$ is a finitely generated module with finite dual Goldie dimension over a right repetitive ring whose matrices are also right repetitive, then End $M$ is semi-local, and if further $M$ is hollow, then End $M$ is local. Example $10(1)$ shows that this result is not true for an arbitrary ring. 
It is well known that a quasi-injective module $M$ has finite Goldie dimension if and only if End $M$ is a semi-perfect ring. Theorem 3 (2) gives an "almost" dual result for quasi-projective modules.

Corollary 4. Let $R$ be a ring and $P$ a right quasi-projective module.

(1) If $P$ has finite dual Goldie dimension, then $\operatorname{End}(P)$ is semi-local.

(2) (Ware, [21]) If $P$ has small radical, then $\operatorname{End}(P)$ is local if and only if $P$ is a local module.

(3) If $P$ has small radical, then $\operatorname{End}(P)$ is semi-local if and only if $P$ has finite dual Goldie dimension.

Proof. To prove (1) observe that if $P$ is a quasi-projective module and $f$ : $P \rightarrow P$ is a surjective endomorphism, then $P \cong X \oplus f(P) \cong X \oplus P$ and hence $P \cong X^{n} \oplus P$ for all $n \geq 1$. If $P$ has finite dual Goldie dimension $k$, then it cannot be a direct sum of more than $k$ proper summands. Thus $X=0$ and we conclude that $f$ is an isomorphism. Now Theorem 3 (2) implies that $\operatorname{End}(P)$ is semi-local.

If $P_{R}$ is local, then Theorem 3 (2) implies immediately that End $P_{R}$ is local. A slight modification in the proof of Proposition 17.19 of [1] yields the converse of this statement. This proves (2).

To prove (3) we only need to show that if $P$ is a quasi-projective module with small radical and whose endomorphism ring is semi-local, then $P$ has finite dual Goldie dimension. Observe that $\bar{P}=P / J(P)$ is quasi-projective as a module over $\bar{R}=R / J(R)$ and $\operatorname{End}\left(\bar{P}_{\bar{R}}\right) \cong \operatorname{End}(P) / J(\operatorname{End} P)$ (cf. [21, Proposition 1.1]) is semi-simple. Hence there exist primitive orthogonal idempotents $e_{1}, \ldots, e_{n}$ in $\operatorname{End}\left(\bar{P}_{\bar{R}}\right)$ such that $1=e_{1}+\cdots+e_{n}$ and $\operatorname{End} e_{i} \bar{P} \cong e_{i} \operatorname{End}(\bar{P}) e_{i}$ is a division ring. Hence $\bar{P}=e_{1} \bar{P} \oplus \cdots \oplus e_{n} \bar{P}$. It follows from (2) that $\left(e_{i} \bar{P}\right)_{\bar{R}}$ is local and hence simple because it has zero radical. This shows that $\bar{P}_{\bar{R}}$ is semi-simple and so $\operatorname{codim}(P)=\operatorname{codim}\left(\bar{P}_{\bar{R}}\right)=n<\infty$.

A right $R$-module $M$ is said to be linearly compact (in the discrete topology), if any system of finitely solvable congruences

$$
x \equiv x_{i} \quad \bmod N_{i}, \quad i \in I, \quad N_{i} \subseteq M, \quad x_{i} \in M,
$$

is solvable. Artinian modules are linearly compact but the importance of linearly compact modules comes from the fact proved by Müller in [18] (see also [22, Corollary 4.2]) that when a ring $R$ has a right Morita duality then the reflexive modules are exactly the right linearly compact ones.

Carl Faith made the conjecture that a linearly compact module should have semi-local endomorphism ring. Since a linearly compact module has both finite dual Goldie dimension (by Zelinsky [23, Proposition 6]) and finite Goldie dimension (by Sandomierski [19, Lemma 2.3] or [22, Propositions 3.4 and 3.3]), Theorem 3 (3) settles the conjecture of Faith in the affirmative.

Corollary 5. Let $R$ be a ring and $M$ a linearly compact right $R$-module. Then the endomorphism ring of $M$ is semi-local.

Right linearly compact rings are semi-perfect ([19, Proposition 2.6 corollary] or [22, Corollary 3.14$]$ ), and since any linearly compact module over a commutative ring is pure-injective, it has semi-perfect endomorphism ring (cf. [12, 
p. 174 and Corollary 8.27]). However in [4, Theorem 3.5] Camps and Menal give an example of a cyclic indecomposable artinian module whose endomorphism ring is semi-local but not local, thus in general it is not true that the endomorphism ring of a linearly compact module is semi-perfect.

We say that a module $M$ satisfies $A B 5^{*}$ if

$$
\bigcap_{i \in I}\left(N+M_{i}\right)=N+\bigcap_{i \in I} M_{i}
$$

for all submodules $N$ and inverse systems of submodules $\left\{M_{i}\right\}_{i \in I}$ of $M$.

Leptin proved that linearly compact modules satisfy $A B 5^{*}([14$, Satz 1$]$ or [22, Corollary 3.9]), but in general a module satisfying $A B 5^{*}$ need not have finite Goldie or dual Goldie dimension (consider for example the $\mathbb{Z}$-module $M=\bigoplus_{p \in P} \mathbb{Z} / p \mathbb{Z}$, where $\mathbb{Z}$ denotes the ring of integers and $P$ is an infinite set of different primes).

Lemma 6. Let $R$ be a ring and $M$ a right $R$-module satisfying $A B 5^{*}$. Then the following statements are equivalent:

(1) Any quotient of $M$ has finite Goldie dimension.

(2) Any submodule of $M$ has finite dual Goldie dimension.

Proof. To prove that (1) implies (2), let $\left\{A_{i}\right\}_{i \in \mathbb{N}}$ be an infinite countable coindependent family of submodules of $M$. Set $P_{i}=\{J \subseteq \mathbb{N} \mid J$ is finite and $i \notin$ $J\}$, for any $J \in P_{i}$ set $M_{J}=\bigcap_{j \in J} A_{j}$. Now $\left\{M_{J}\right\}$ is an inverse subsystem of submodules of $M$. Applying $A B 5^{*}$ we have

$$
\bigcap_{J \in P_{i}}\left(A_{i}+M_{J}\right)=A_{i}+\bigcap_{J \in P_{i}} M_{J}
$$

By the definition of coindependence $A_{i}+M_{J}=M$, thus for any $i \in \mathbb{N}, A_{i}+$ $\bigcap_{J \in P_{i}} M_{J}=A_{i}+\bigcap_{j \neq i} A_{j}=M$. This proves that the image of the natural morphism $M \longrightarrow \prod_{i \in \mathbb{N}} M / A_{i}$ contains an infinite direct sum, which contradicts (1). Hence $M$ has no infinite coindependent families of submodules. Since submodules of modules with $A B 5^{*}$ also have this property, the result follows.

It is very easy to see that (2) always implies (1).

If $M$ is a right $R$-module, we denote by $\mathscr{S}(M)$ the set of non-isomorphic simple images of submodules of $M$.

Corollary 7. If $R$ is a ring and $M$ a right $R$-module satisfying $A B 5^{*}$ such that $\mathscr{S}(M)$ is finite, then $\operatorname{End}(M)$ is semi-local.

Proof. Lemonnier in [13, Lemme 2] proves that if $M$ is a right $R$-module satisfying $A B 5^{*}$ such that $\mathscr{S}(M)$ is finite, then any quotient of $M$ has finite Goldie dimension. Now the result follows from Lemma 6 and Theorem 3(3).

The result of Corollary 7 does not include all linearly compact modules, since there exist examples of linearly compact modules such that $\mathscr{S}(M)$ is not finite-see [8, Examples 3 and 4].

The next result enables us to show that over a commutative ring a module satisfying $A B 5^{*}$ satisfies the conclusions of Theorem 2 .

If $M$ is a right $R$-module and $A$ is a subset of $M$, put $r_{R}(A)=\{r \in R \mid$ $A r=0\}$. 
Lemma 8. Let $R$ be a commutative ring and $M$ an $R$-module such that for any $x \in M, R / r_{R}(x)$ is a semi-perfect ring. Then $M=\bigoplus_{i \in I} M_{i}$ where $M_{i}$ is a module over a local ring $R_{i}$ and $\operatorname{End}_{R}(M) \cong \prod_{i \in I} \operatorname{End}_{R_{i}}\left(M_{i}\right)$.

Proof. For any $S_{i} \in \mathscr{S}(M)$ consider $E\left(S_{i}\right)$, the injective hull of the simple module $S_{i}$, and set

$$
M_{i}=\left\{x \in M \mid \operatorname{Hom}\left(x R, E\left(S_{j}\right)\right)=0 \text { for all } j \neq i\right\} .
$$

It is easy to see that

$M_{i}=\left\{x \in M \mid R / r_{R}(x)\right.$ is a local ring with simple module $\left.S_{i}\right\} \cup\{0\}$.

We prove first that $M_{i}$ is a submodule of $M$. Since $E\left(S_{i}\right)$ is injective it is clear that $x r \in M_{i}$ whenever $x \in M_{i}$ and $r \in R$. Let $x$ and $y$ be non-zero elements of $M_{i}$ and let $f:(x+y) R \longrightarrow E\left(S_{j}\right), j \neq i$, be any morphism. Then $f((x+y) R) r_{R}(x)=0$ and so $\operatorname{im} f$ is an $R / r_{R}(x)$-module. But $x \in M_{i}$ and by the definition of $M_{i}, R / r_{R}(x)$ is a local ring with simple module $S_{i}$, thus im $f=0$ and we conclude that $x+y \in M_{i}$.

It is clear that $\left\{M_{i}\right\}$ form a family of independent submodules of $M$ such that $\operatorname{Hom}\left(M_{i}, M_{j}\right)=0$ for $i \neq j$, and since for any $x \in M, x R \cong R / r_{R}(x R)$ is a commutative semi-perfect ring, we deduce that $M=\bigoplus M_{i}$.

Consider $S_{i} \in \mathscr{S}(M), S_{i} \cong R / P_{i}$, for a suitable maximal ideal $P_{i}$ of $R$. To finish the proof, we show that $M_{i}$ is an $R_{P_{i}}$-module and $\operatorname{End}_{R}\left(M_{i}\right)=$ $\operatorname{End}_{R_{P_{i}}}\left(M_{i}\right)$. The definition of $M_{i}$ implies that $r_{R}(x) \subseteq P_{i}$ for any $0 \neq x \in M_{i}$, hence for any $a \in R \backslash P_{i}$ multiplication by $a$ induces an injective $R / r_{R}(x)$ endomorphism $f$ of $x R$, and since $a$ is a unit in $R / r_{R}(x), f$ is also surjective. We conclude that $M_{i}$ is an $R_{P_{i}}$-module, and as it is clear that $\operatorname{End}_{R}\left(M_{i}\right)=$ $\operatorname{End}_{R_{P_{t}}}\left(M_{i}\right)$, the proof of the lemma is complete.

Corollary 9. Let $R$ be a commutative ring and $M$ a module satisfying $A B 5^{*}$. Then $\operatorname{End}(M)$ is a product of semi-local rings, 1 is in the stable range of $\operatorname{End}(M)$, and $M$ cancels from direct sums and satisfies the $n$th root uniqueness property. Proof. If $M$ is a module satisfying $A B 5^{*}$, then by [13, Proposition 4] for any $x \in M, x R \cong R / r_{R}(x)$ is a semi-perfect ring. Apply Lemma 8 and Corollary 7 to conclude that $\operatorname{End}(M)$ is a product of semi-local rings. Thus by Theorem 2, 1 is in the stable range of $\operatorname{End}(M)$, and by [6, Theorem 2] $M$ cancels from direct sums.

Theorem 2 implies that $M$ is a direct sum of modules that cancel from direct sums and satisfy the $n$th root uniqueness property, so $M$ itself satisfies the $n$th root uniqueness property.

Remark. It is easy to see that the rings such that every right ideal and every left ideal is an annihilator satisfy $A B 5^{*}$ (on both sides). These rings were studied by Hajarnavis and Norton in [11]. Lemonnier's results in [13] give alternative and shorter proofs to Theorems 3.9 and 5.3 in the Hajarnavis and Norton paper, who also show that if $R$ is a ring such that any right and left ideal is an annihilator, then $R / \bigcap_{n=1}^{\infty} J(R)^{n}$ is a noetherian ring; it is easy to see that their proof also works for rings satisfying $A B 5^{*}$. Müller in [17] or [22, Lemma 17.1] proves that if $R$ is a right linearly compact ring, then $R / \bigcap_{n=1}^{\infty} J(R)^{n}$ is a right noetherian ring and in his proof only right $A B 5^{*}$ is used. In [15] Menini proved that a twosided noetherian and right linearly compact ring satisfies that $\bigcap_{n=1}^{\infty} J(R)^{n}=0$ 
(see also [22, Corollary 17.5]). Again in Menini's proof the only property used of right linear compactness is right $A B 5^{*}$.

Thus if $R$ is a ring satisfying right $A B 5^{*}$, then:

(1) (Müller [17]) $R / \bigcap_{n=1}^{\infty} J(R)^{n}$ is a noetherian ring.

(2) (Menini [15]) If $R$ is right and left noetherian, then $\bigcap_{n=1}^{\infty} J(R)^{n}=0$.

In [16, Question 11, p. 106] Mohamed and Müller ask for examples of local modules whose endomorphism ring is not local. In [4, Theorem 3.5] Camps and Menal construct examples of indecomposable artinian cyclic modules $M$ whose endomorphism ring is semi-local but not local. It is easy to see that in some of these examples $M$ is also a local module. The next example, patterned after Camps and Menal techniques, shows that any ring that can be embedded in a local ring can be realized as the endomorphism ring of a local module.

Until now all the examples we have given of modules with semi-local endomorphism ring (except perhaps injective modules with finite Goldie dimension) have finite dual Goldie dimension. It is clear that if $R$ is commutative any cyclic module with semi-local endomorphism ring should have finite dual Goldie dimension but, as the next example shows, this is not true over arbitrary rings.

Example 10. (1) Let $R$ be a ring that can be embedded in a local ring $S$. Then $R$ can be realized as the endomorphism ring of a local module.

(2) There exist cyclic modules with infinite dual Goldie dimension whose endomorphism ring is semi-local.

Proof. Let $R \subseteq S$ be an embedding of rings, and consider the $(S, R)$-bimodule $M=\operatorname{Hom}_{R}\left({ }_{R} S,{ }_{R} S / R\right)$ and the sub-bimodule $N=\{f \in M \mid f(R)=0\}$. Let $T$ be the ring $T=\left(\begin{array}{ll}S & M \\ 0 & R\end{array}\right)$ and consider the right ideal $I=\left(\begin{array}{ll}0 & N \\ 0 & R\end{array}\right)$ of $T$. The idealizer of $I$ is $I^{\prime}=\left(\begin{array}{ll}R & N \\ 0 & R\end{array}\right)$ because an element $\left(\begin{array}{l}s f \\ 0 r\end{array}\right) \in I^{\prime}$ if and only if $s N \subseteq N$ and $f R \subseteq N$ which implies that $s \in R$ and $f \in N$. Thus $\operatorname{End}_{T}(T / I)=I^{\prime} / I=R$.

To prove (1) assume that $S$ is a local ring. The proper right ideals of $T$ containing $I$ are of the form $\left(\begin{array}{ll}J & K \\ 0 & R\end{array}\right)$, where $J$ is a right ideal of $S$ different from $S$, and $K$ is a sub-bimodule of $M$ containing $N$. Since $J$ is a small submodule of $S$, every proper submodule of $T / I$ is small. Hence $T / I$ is a local right $T$-module with endomorphism ring $R$.

To prove (2) assume that $R$ is semi-local and $S$ is not, thus $S$ has an infinite co-independent family $\left\{A_{i}\right\}_{i \in \mathbb{N}}$ of right ideals. The right ideals of $T$, $\left\{\left(\begin{array}{cc}A_{i} & M \\ 0 & R\end{array}\right)\right\}_{i \in \mathbb{N}}$, will give an infinite family of coindependent submodules of $T / I$. Thus $T / I$ has infinite dual Goldie dimension but its endomorphism ring is the semi-local ring $R$.

\section{ACKNOWLEDGMENT}

This work was done in the Mathematics Department at Rutgers University. Both authors wish to thank their host for its hospitality and mention in particular Professor Carl Faith whose conjecture that linearly compact modules should have semi-local endomorphism ring motivated this paper. The research of the first author was supported by a postdoctoral fellowship from the Ministerio de Educación y Ciencia of Spain and the Fulbright Foreign Scholarship Board and 
a grant from the DGYCIT(Spain). The research of the second author was partially supported by a grant from the University Research Board of the American University of Beirut.

\section{REFERENCES}

1. F. W. Anderson and K. R. Fuller, Rings and categories of modules, 2nd ed., Graduate Texts in Math., Springer-Verlag, New York, 1992.

2. H. Bass, K-theory and stable algebra, Inst. Hautes Études Sci. Publ. Math. 22 (1964), 5-60.

3. R. Camps and W. Dicks, On semi-local rings, Israel J. Math. 81 (1993), 203-211.

4. R. Camps and P. Menal, Power cancellation for Artinian modules, Comm. Algebra 19 (1991), 2081-2095.

5. F. Dischinger, Sur les anneaux fortement $\pi$-réguliers, C. R. Acad. Sci. Paris Sér. A 283 (1976), 571-573.

6. E. G. Evans, Krull-Schmidt and cancellation over local rings, Pacific J. Math. 46 (1973), 115-121.

7. A. Facchini, D. Herbera, L. S. Levy, and P. Vamos, Krull-Schmidt fails for Artinian modules, Proc. Amer. Math. Soc. (to appear).

8. L. Fuchs, Torsion preradicals and ascending Loewy series of modules, J. Reine Angew. Math. 239 (1970), 169-179.

9. K. R. Goodearl, Surjective endomorphism of finitely generated modules, Comm. Algebra 15 (1987), 589-609.

10. A. Hanna and A. Shamsuddin, Duality in the category of modules. Applications, Algebra Ber., vol. 49, Fishcer, München, 1984.

11. C. R. Hajarnavis and N. C. Norton, On dual rings and their modules, J. Algebra 93 (1985), 253-266.

12. C. Jensen and H. Lenzing, Model theoretic algebra, Gordon and Breach, New York, 1989.

13. B. Lemonnier, $A B 5^{*}$ et la dualité de Morita, C. R. Acad. Sci. Paris Sér. A 289 (1979), 47-50.

14. H. Leptin, Linear kompakte Moduln und Ringe I, Math. Z. 62 (1955), 241-267.

15. C. Menini, Jacobson's conjecture, Morita duality and related questions, J. Algebra 103 (1986), 638-655.

16. S. H. Mohamed and B. J. Müller, Continuous and discrete modules, London Math. Soc. Lecture Note Ser., vol. 147, Cambridge Univ. Press, Cambridge, 1990.

17. B. J. Müller, On Morita duality, Canad. J. Math. 26 (1969), 1338-1347.

18. L Linear compactness and Morita duality, J. Algebra 16 (1970), 60-66.

19. F. Sandomierski, Linearly compact modules and Morita duality, Ring Theory, Conference on Ring Theory (Park City, Utah) (R. Gordon, ed.), Academic Press, New York and London, 1972, pp. 333-346.

20. K. Varadarajan, Dual Goldie dimension, Comm. Algebra 7 (1979), 565-610.

21. R. Ware, Endomorphism rings of projective modules, Trans. Amer. Math. Soc. 155 (1971), 233-256.

22. W. Xue, Rings with Morita duality, Lecture Notes in Math., vol. 1523, Springer-Verlag, New York, 1992.

23. D. Zelinsky, Linearly compact modules and rings, Amer. J. Math. 75 (1953), 79-90.

Department of Mathematics, Rutgers University, New Brunswick, New Jersey 08903 Current address: Departament de Matemàtiques, Universitat Autònoma de Barcelona, 08193 Bellatera, Barcelona, Spain

E-mail address: dolors@mat uab.es

Department of Mathematics, American University of Beirut, Beirut, Lebanon

E-mail address: ahmadelayla.aub.ac.lb 